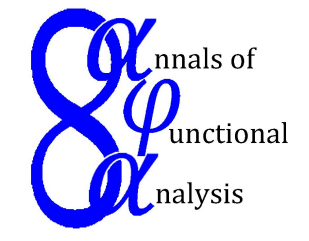

Ann. Funct. Anal. 5 (2014), no. 2, 47-52

$\mathscr{A}$ NNALS OF $\mathscr{F}$ UNCTIONAL $\mathscr{A}$ NALYSIS

ISSN: 2008-8752 (electronic)

URL:www.emis.de/journals/AFA/

\title{
COMMUTATORS OF TWO COMPRESSED SHIFTS AND THE HARDY SPACE ON THE BIDISC
}

\author{
TAKAHIKO NAKAZI \\ Dedicated to Professor Tsuyoshi Ando on his eightieth birthday \\ Communicated by S. Barza
}

\begin{abstract}
For a subset $E$ of the bidisc $D^{2}, M=\left\{f \in H^{2}\left(D^{2}\right): f=0\right.$ on $E\}$ and $N$ is the orthogonal complement of $M$ in $H^{2}\left(D^{2}\right)$ where $H^{2}\left(D^{2}\right)$ is the two variable Hardy space on $D^{2}$. We describe the finite rank commutants of the restricted shifts $S_{z}$ and $S_{w}$ on $N$ when $E$ satisfies some natural condition. Moreover we give a sufficient condition for that the Pick interpolation is possible.
\end{abstract}

\section{INTRODUCTION}

For $1 \leq p \leq \infty, H^{p}(D)$ denotes the one variable Hardy space on the open unit disc $D$ in $\mathbb{C}$ and $H^{p}\left(D^{2}\right)$ denotes the two variable Hardy space on $D^{2}=D \times D$. Let $m$ be the normalized Lebesgue measure on $T^{2}=T \times T$ when $T=\partial D$. Each $f$ in $H^{2}\left(D^{2}\right)$ has a radial limit $f^{*}$ defined on $T^{2}$ a.e. $m$ and let $H^{p}\left(T^{2}\right)=\left\{f^{*}: f \in\right.$ $\left.H^{p}\left(D^{2}\right)\right\}$. Then $H^{p}\left(T^{2}\right)$ is a Banach space in $L^{p}\left(T^{2}\right)=L^{p}\left(T^{2}, m\right)$. It is known that $H^{p}\left(D^{2}\right)$ is isometrically isomorphic to $H^{p}\left(T^{2}\right)$. These facts are shown in [5]. $z$ and $w$ are the coordinates of the functions on $\mathbb{C}^{2}=\mathbb{C} \times \mathbb{C}$

A closed subspace $M$ of $H^{2}\left(D^{2}\right)$ is said to be an invariant subspace if $z M \subset M$ and $w M \subset M$. Let $N$ be the orthogonal complement of $M$ in $H^{2}\left(D^{2}\right)$. In this paper, we assume that

$$
M=\left\{f \in H^{2}\left(D^{2}\right): f=0 \text { on } E\right\}
$$

for a subset $E$ of $D^{2}$. Then $N$ is the closed linear span of $\left\{(1-\bar{a} z)^{-1}(1-\bar{b} w)^{-1}\right.$ : $(a, b) \in E\}$. Hence $N \cap H^{\infty}\left(D^{2}\right)$ is dense in $N$.

Date: Received: May 22, 2013; Revised: August 5, 2013; Accepted: October 7, 2013.

2010 Mathematics Subject Classification. Primary 47B32; Secondary 32A35.

Key words and phrases. Rrestricted shift, commutant, finite rank, bidisc. 
For a function $\psi$ in $H^{2}\left(D^{2}\right)$, put

$$
S_{\psi} f=P^{N}(\psi f) \quad\left(f \in N \cap H^{\infty}\left(D^{2}\right)\right)
$$

where $P^{N}$ is the orthogonal projection onto $N$. We do not know whether $S_{\psi}$ is bounded or not. The following question is natural. Does there exist a function $\phi$ in $H^{\infty}\left(D^{2}\right)$ such that $S_{\psi}=S_{\phi}$ when $S_{\psi}$ is bounded? However this question is answered negatively [2]. In this paper we consider the following problem.

Problem 1.1. Let $\psi$ be a function in $H^{2}\left(D^{2}\right)$. If $S_{\psi}$ is of finite rank then does there exist a function $\phi$ in $H^{\infty}\left(D^{2}\right)$ such that $S_{\psi}=S_{\phi}$ ?

If $A$ is a bounded linear operator on $N$ such that $A S_{z}=S_{z} A$ and $A S_{w}=S_{w} A$ then it easy to see that $A=S_{\psi}$ on $N \cap H^{\infty}\left(D^{2}\right)$ for some $\psi$ in $H^{2}\left(D^{2}\right)$. Conversely if $A=S_{\psi}$ on $N \cap H^{\infty}\left(D^{2}\right)$ for some $\psi$ in $H^{2}\left(D^{2}\right)$ then $A S_{z}=S_{z} A$ and $A S_{w}=S_{w} A$ on $N \cap H^{\infty}\left(D^{2}\right)$. For one variable $H^{2}(D)$, this is in [6]. The same proof is valid in two variable $H^{2}\left(D^{2}\right)$. Now it follows that $A S_{z}=S_{z} A$ and $A S_{w}=S_{w} A$ on $N$ because $N \cap H^{\infty}\left(D^{2}\right)$ is dense in $N$. Hence if the problem above can be solved positively then finite rank operators of commutants of $S_{z}$ and $S_{w}$ are described.

For an arbitrary invariant subspace $M$, Sarason [6] solved this problem in 1967 for one variable $H^{\infty}(D)$ without the finite rank condition. Hence if $\psi$ is one variable then the above problem can be solved.

Suppose that $\left\{\zeta_{j}\right\}_{j=1}^{n}=\left\{\left(a_{j}, b_{j}\right)\right\}_{j=1}^{n}$ is in $D^{2}$ and $\left\{\eta_{j}\right\}_{j=1}^{n}$ is in $\mathbb{C}$. When $\zeta=$ $(z, w), k_{\zeta_{j}}(\zeta)$ denotes the reproducing kernel of $\zeta_{j}$ for $H^{2}\left(D^{2}\right)$, that is, $k_{\zeta_{j}}(\zeta)=$ $\left(1-\overline{a_{j}} z\right)^{-1}\left(1-\overline{b_{j}} w\right)^{-1}$. In this paper we consider the following problem.

Problem 1.2. If the Pick matrix

$$
\left[\left(1-\eta_{i} \bar{\eta}_{j}\right) k_{\zeta_{j}}\left(\zeta_{i}\right)\right] \geq 0 \quad(1 \leq j \leq n)
$$

then does there exist a function $\phi$ in $H^{\infty}\left(D^{2}\right)$ such that $\|\phi\|_{\infty} \leq 1$ and $\phi\left(\zeta_{j}\right)=$ $\eta_{j}(1 \leq j \leq n) ?$

In Problem 1.2, it is easy to see the converse is valid. Unfortunately it is known [1] that Problem 1.2 can be solved negatively in general. Hence we will consider it in some special case.

G. Pick solved this problem in 1916 for one variable $H^{\infty}(D)$. Hence if $\zeta_{j}=$ $\left(a_{j}, b_{j}\right)(1 \leq j \leq n)$ and $b_{1}=b_{2}=\cdots=b_{n}$ then the above problem can be solved. It should be noted that Agler and $\mathrm{M}^{c}$ Carthy [1] solved a two variable interpolation problem in a different form from the above problem.

For a subset $E$ of $D^{2}$, put $E_{a}=\{b \in D:(a, b) \in E\}$ and $E_{\cdot b}=\{a \in D$ : $(a, b) \in E\}$.

\section{Problem 1}

In this section we solve Problem 1.1 under some condition. We do not use Sarason's generalized interpolation theorem [6]. For $0<p \leq \infty$, if $E \subset D^{2}$ and $c \in E$ then put

$$
\rho_{p}(c)=\rho_{p}(c, E)=\sup \left\{|f(c)|: f \in H^{p}\left(D^{2}\right),\|f\|_{p} \leq 1 \text { and } f=0 \text { on } E \backslash\{c\}\right\} .
$$


In general, it may not be true that $\rho_{p}(a)=\rho_{q}(a)$ for $p \neq q$. If $E$ is a finite set, then $\rho_{p}(c)>0$ for $0<p \leq \infty$. But in general it may happen that $\rho_{p}(c)=0$ for $0<p \leq \infty$. In fact, suppose $E=\{(0, w): w \in D\}$ then $\rho_{p}(c)=0$ for any $c \in E$. There exists a set $E$ in $D^{2}$ such that $\rho_{\infty}(c)=0$ and $\rho_{2}(c)>0$. In fact, we can find such a set in $\left\{\left(a_{j}, a_{j}\right) \in D^{2}: \sum_{j=1}^{\infty}\left(1-\left|a_{j}\right|^{2}\right)=\infty\right\}$. For $\left\{f(z, z): f \in H^{2}\left(D^{2}\right)\right\}$ is a one variable Bergman space $L_{a}^{2}$ (see [5, p.53]) and $\left\{f(z, z): f \in H^{\infty}\left(D^{2}\right)\right\}$ is just $H^{\infty}(D)$. It is known that there is a nonzero function in $L_{a}^{2}(D)$ whose zero set does not satisfy a Blaschke condition.

Theorem 2.1. Suppose $M=\left\{f \in H^{2}\left(D^{2}\right): f=0\right.$ on $\left.E\right\}$ for a subset $E$ of $D^{2}$ and $\rho_{\infty}(a)>0$ when $\rho_{2}(a)>0$ for a in $E$. If $\psi$ is a function in $H^{2}\left(D^{2}\right)$ such that $S_{\psi}$ is of finite rank on $N$ the orthogonal complement of $M$, then there exists a function $\phi$ in $H^{\infty}\left(D^{2}\right)$ such that $S_{\psi}=S_{\phi}$.

Proof. Suppose $S_{\psi}$ is of finite rank $n$. If $n=0$ then the theorem is clear and so we may assume $n>0$. Then the range of $S_{\psi}$ is of $n$ dimension and so there exist $k_{j}$ in $N(1 \leq j \leq n)$ such that $S_{\psi} N=$ the linear $\operatorname{span}\left\langle k_{1}, \cdots, k_{n}\right\rangle$ of $k_{1}, \cdots, k_{n}$. Since $S_{z}$ and $S_{w}$ commute with $S_{\psi}$ (see Introduction), $\left\langle k_{1}, \cdots, k_{n}\right\rangle$ is an invariant subspace of $S_{z}$ and $S_{w}$. Hence there exist one variable minimal polynomials $p=p(z)$ and $q=q(w)$ such that $p\left(S_{z}\right) k_{j}=S_{p} k_{j}=0$ and $q\left(S_{w}\right) k_{j}=S_{q} k_{j}=0$ for $1 \leq j \leq n$. Therefore $p k_{j}$ and $q k_{j}$ belong to $M$ for $1 \leq j \leq n$. Then we may assume zeros of $p$ and $q$ are all simple by the definition of $M$. Hence for all $f$ in $N p S_{\psi} f=q S_{\psi} f=0$ and so $p \psi f=q \psi f=0$ on $E$. For each $(x, y) \in E$, there is a function $F$ in $H^{2}$ with $F(x, y) \neq 0$. Put $f=P^{N} F$ then $f(x, y)=F(x, y)$ because $\left(I-P^{N}\right) F=0$ on $E$. Therefore $p(x) \psi(x, y)=q(y) \psi(x, y)=0$ for any $(x, y) \in E$. Thus

$$
\psi=0 \text { on } E \backslash\{(x, y) \in E: p(x)=q(y)=0\} .
$$

Hence there exist $\left(a_{1}, b_{1}\right), \cdots,\left(a_{m}, b_{m}\right)$ in $E$ such that $\psi=0$ on $E \backslash \bigcup_{j=1}^{m}\left(a_{j}, b_{j}\right)$. Since $p$ and $q$ are minimal polynomials of $S_{z}$ and $S_{w}$, respectively, $\psi\left(a_{j}, b_{j}\right) \neq 0$ if $p\left(a_{j}\right)=q\left(b_{j}\right)=0$. For each $1 \leq j \leq m$, there exists a $f_{j} \in H^{\infty}\left(D^{2}\right)$ such that $f_{j}\left(a_{j}, b_{j}\right)=1$ and $f_{j}\left(a_{\ell}, b_{\ell}\right)=0$ if $j \neq \ell$. Put $\psi_{j}=f_{j} \psi$ then $\psi_{j}\left(a_{j}, b_{j}\right)=$ $\psi\left(a_{j}, b_{j}\right) \neq 0$ and so $\rho_{2}\left(\left(a_{j}, b_{j}\right)\right)>0$. Since $\rho_{\infty}\left(\left(a_{j}, b_{j}\right)\right)>0$ for $1 \leq j \leq m$ by the hypothesis, there exist $h_{j}$ in $H^{\infty}\left(D^{2}\right)$ such that

$$
h_{j}\left(a_{j}, b_{j}\right) \neq 0 \text { and } h_{j}=0 \text { on } E \backslash\left(a_{j}, b_{j}\right) .
$$

Hence there exist $\alpha_{1}, \cdots, \alpha_{n}$ in $\mathbb{C}$ such that $\psi-\sum_{j=1}^{m} \alpha_{j} h_{j} \in M$. Put $\phi=$ $\sum_{j=1}^{m} \alpha_{j} h_{j}$ then $\phi$ belongs to $H^{\infty}\left(D^{2}\right)$ and $S_{\phi}=S_{\psi}$.

Theorem 2.2. Suppose $M=\left\{f \in H^{2}\left(D^{2}\right): f=0\right.$ on $\left.E\right\}$ for a subset $E$ of $D^{2}$ and $\rho_{2}(a)=0$ for any a in $E$. If $\psi$ is a function in $H^{2}\left(D^{2}\right)$ such that $S_{\psi}$ is of finite rank on $N$, then there exists a function $\phi$ in $H^{\infty}\left(D^{2}\right)$ such that $S_{\psi}=S_{\phi}=0$.

Proof. If $S_{\psi}=0$, put $\phi \equiv 0$ then $S_{\psi}=S_{\phi}$ and $S_{\phi}=0$. Hence we assume $S_{\psi} \neq 0$. By the proof of Theorem 2.1, there exist minimal polynomials $p=p(z)$ and $q=q(w)$ which are one variable polynomials of degree $\ell \leq n$ such that $p \psi f \in M$ and $q \psi g \in M$ for any $f$ and $g$ in $N \cap H^{\infty}\left(D^{2}\right)$. Then the zeros of $p$ (and $q$ ) are all simple and the degree of $p$ (and $q$ ) is minimal, respectively. By 
the proof of Theorem 2.1, there exists $\left(a_{1}, b_{1}\right), \cdots,\left(a_{m}, b_{m}\right)$ in $E$ such that $\psi=0$ on $E \backslash \bigcup_{j=1}^{m}\left(a_{j}, b_{j}\right)$ and $p\left(a_{j}\right)=0$ or $q\left(b_{j}\right)=0(1 \leq j \leq m)$. Let $p_{t}=p /\left(z-a_{t}\right)$ and $q_{t}=q /\left(z-b_{t}\right)$, then by the minimality of $p$ and $q$ there exist $f_{0}$ and $g_{0}$ in $N \cap H^{\infty}\left(D^{2}\right)$ such that $p_{t} \psi f_{0} \notin M$ and $q_{t} \psi g_{0} \notin M$. Hence $p_{t} \psi \notin M$ and $q_{t} \psi \notin M$ because $z M \subseteq M$ and $w M \subseteq M$. Since $\psi=0$ on $E \backslash \bigcup_{j=1}^{m}\left(a_{j}, b_{j}\right)$ and $p_{t}\left(a_{j}\right) \neq 0$ or $q_{t}\left(b_{j}\right) \neq 0$ for $j \neq t, p_{t} \psi=q_{t} \psi=0$ on $E \backslash\left(a_{t}, b_{t}\right)$. Hence $\left(p_{t} \psi\right)\left(a_{t}, b_{t}\right) \neq 0$ and $\left(q_{t} \psi\right)\left(a_{t}, b_{t}\right) \neq 0$ because $p_{t} \psi \notin M$ and $q_{t} \psi \notin M$. This contradicts $\rho_{2}(a)=0$ for any a in $E$.

When $E$ does not have any isolated points, if $\psi$ is a function in $H^{2}\left(D^{2}\right)$ such that $S_{\psi}$ is of finite rank on $N$ then there exists a function $\phi$ in $H^{\infty}\left(D^{2}\right)$ such that $S_{\psi}=S_{\phi}=0$.

Suppose $M=\left\{f \in H^{2}\left(D^{2}\right): f=0\right.$ on $\left.E\right\}$. Then it is known [5, Theorem 4.1.1] that there exists an example $E \subset D$ such that $M \cap H^{\infty}\left(D^{2}\right)=\{0\}$. Then it is easy to see that there exists $a$ in the example such that $\rho_{2}(a)>0$ and $\rho_{\infty}(a)=0$. When $E$ is an interpolation sequence of $H^{\infty}\left(D^{2}\right)$, if $\rho_{2}(a)>0$ then $\rho_{\infty}(a)>0$ for any $a$ in $E$.

It is easy to see that if there exists $a \in E$ such that $\rho_{2}(a)>0$ then there exists $\psi$ in $H^{2}\left(D^{2}\right)$ and $\operatorname{dim} S_{\psi}\left(N \cap H^{\infty}\left(D^{2}\right)\right)=1$. Conversely if $S_{\psi}$ is of rank one and $\psi$ is in $H^{2}\left(D^{2}\right)$ then the proof of Theorem 2.1 shows there exists $a \in E$ such that $\rho_{2}(a)>0$.

\section{Problem 2}

Let $\left\{a_{j}\right\}_{j=1}^{n}$ and $\left\{b_{j}\right\}_{j=1}^{m}$ be $n$ and $m$ distinct points in $D$, respectively. Put $\zeta_{i j}=\left(a_{i}, b_{j}\right)$ for $1 \leq i \leq n$ and $1 \leq j \leq m$. Then $\zeta_{i j} \neq \zeta_{\ell k}$ if $(i, j) \neq(\ell, k)$. Let $\left\{u_{j}\right\}_{j=1}^{n}$ and $\left\{v_{j}\right\}_{j=1}^{m}$ be in $\mathbb{C}$. Put $w_{i j}=u_{i} v_{j}, x=\max u_{i}$ and $y=\max v_{j}$ with $x y \leq 1$. Under these notations, it is easy to see the following simple result by the original Pick's theorem for the disc.

Let $\Lambda$ be a set $\{(i, j): 1 \leq i \leq n, 1 \leq j \leq m\}$. There exist $f$ in $H^{\infty}(D, z)$ with $\|f\|_{\infty} \leq x$ and $g$ in $H^{\infty}(D, w)$ with $\|g\|_{\infty} \leq y$ such that $F(z, w)=f(z) g(w)$ and $F\left(\zeta_{i j}\right)=f\left(a_{i}\right) g\left(b_{j}\right)=u_{i} v_{j}=w_{i j}((i, j) \in \Lambda)$ and $\|F\|_{\infty} \leq 1$ if and only if

$$
\left[\frac{x-u_{s} \overline{u_{t}}}{1-\bar{a}_{t} a_{s}}\right]_{n \times m} \geq 0 \text { and }\left[\frac{y-v_{s} \bar{v}_{t}}{1-\bar{b}_{t} b_{s}}\right]_{m \times m} \geq 0 .
$$

\section{Not NECESSARY FINITE RANK CASE}

The condition in Theorem 4.1 satisfies one in Theorem 1. We use the generalized interpolation theorem of Sarason [6] for the proof. We consider a not necessary finite rank case. Moreover we consider 'Conjecture' in the previous paper [4]. Let $\mathcal{A}$ be the weak closed commutative Banach algebra generated by $S_{z}, S_{w}$ and the identity operator and let $\mathcal{A}^{\prime}$ denote the commutant of $\mathcal{A}$ in the Banach algebra of all bounded linear operators in $N$. 'Conjecture' is $\mathcal{A}=\mathcal{A}^{\prime}$, that is, $\mathcal{A}^{\prime}=\left\{S_{\phi}: \phi \in H^{\infty}\left(D^{2}\right)\right\}$. 
Theorem 4.1. Suppose $E=\bigcup_{j=1}^{\infty} E_{a_{j}}$ and $\left\{a_{j}\right\}$ is uniformly separated in $D$ and $M=\left\{f \in H^{2}\left(D^{2}\right): f=0\right.$ on $\left.E\right\}$. If $S_{\psi}$ is bounded and $\psi$ is in $H^{2}\left(D^{2}\right)$ then for any finite $p$ there exists a function $\phi$ in $H^{p}\left(D^{2}\right)$ such that $S_{\psi}=S_{\phi}$.

Proof. By hypothesis, $E=\bigcup_{j=1}^{\infty} E_{a_{j}}$. and $\left\{a_{j}\right\}$ is uniformly separated in $D$. Since $\psi \in H^{2}\left(D^{2}\right), \psi_{j}(w)=\psi\left(a_{j}, w\right) \in H^{2}(D, w)$. If $E_{a_{j}}$. does not satisfy a Blaschke condition then $E_{a_{j}} .=\left\{a_{j}\right\} \times \bar{D}$ and so $M \subset\left(z-a_{j}\right) H^{2}\left(D^{2}\right)$. For if $f$ is in $M$ then $f\left(a_{j}, w\right)=0$ on $E_{a_{j}}$. and so $f\left(a_{j}, w\right) \equiv 0$ on $\bar{D}$. Hence $N \supset\left(1-\bar{a}_{j} z\right)^{-1} H^{2}(D, w)$ and so it is clear that $\psi_{j}(w) \in H^{\infty}(D, w)$ for $1 \leq j \leq m$. Then put $\phi_{j}=$ $\psi_{j}$. If $E_{a_{j}}$. satisfies a Blaschke condition then $E_{a_{j}} .=\left\{a_{j}\right\} \times\left\{b_{j 1}, b_{j 2}, \cdots\right\}$ and $\sum_{\ell=1}^{\infty}\left(1-\left|b_{j \ell}\right|\right)<\infty$. Put $M_{j}=\left\{f \in H^{2}\left(D^{2}\right): f\left(a_{j}, b_{j \ell}\right)=0\right.$ for $\left.\ell=1,2, \cdots\right\}$ then $M_{j} \supset M$ and $N_{j} \subset N$ if $N_{j}=H^{2}\left(D^{2}\right) \ominus M_{j}$. Moreover $N_{j}$ is the closed linear span of

$$
\left\{\frac{c_{\ell}}{\left(1-\overline{a_{j}} z\right)\left(1-\overline{b_{j \ell}} w\right)}: \ell=1,2, \cdots\right\} .
$$

Hence $S_{\psi}^{*}\left|N_{j}=S_{\psi_{j}}^{*}\right| N_{j}$ and so there exists a $\phi_{j} \in H^{\infty}(D, w)$ such that $\psi_{j}-\phi_{j} \in M_{j}$ by the theorem of Sarason.

Considering $H^{p}\left(D^{2}\right) \supset H^{\infty}\left(D, H^{p}\right)$ for any $1 \leq p<\infty$, the vector valued Hardy space on $D$, we can apply a theorem of Aron, Globevnik and Schottenloher [3] because $\left\{a_{j}\right\}$ is uniformly separated. There exists a function $\tilde{\phi} \in H^{\infty}\left(D, H^{p}\right)$ such that $\tilde{\phi}\left(a_{j}\right)=\phi_{j}(w)(j=1,2, \cdots)$. Since we can write $\tilde{\phi}(z)=\phi(z, w) \in$ $H^{p}\left(D^{2}\right), \phi\left(a_{j}, w\right)=\phi_{j}(w)(j=1,2, \cdots)$. Then $\psi-\phi$ belongs to $M$ and so $S_{\psi}=S_{\phi}$.

If $q_{1}=q_{1}(z)$ and $q_{2}=q_{2}(w)$ are one variable Blaschke products with the zero sets $\left\{a_{j}\right\}$ and $\left\{b_{j}\right\}$, respectively. Moreover if we assume that $a_{i} \neq a_{j}(i \neq j)$ and $b_{i} \neq b_{j}(i \neq j)$ then $M=q_{1} H^{2}\left(D^{2}\right)+q_{2} H^{2}\left(D^{2}\right)=\left\{f \in H^{2}\left(D^{2}\right): f\left(a_{j}, b_{j}\right)=\right.$ $01 \leq j<n\}$ where $n$ may be $\infty$. Hence $N=\left(H^{2}(D, z) \ominus q_{1} H^{2}(D, z)\right) \otimes$ $\left(H^{2}(D, w) \ominus q_{2} H^{2}(D, w)\right)$. Now we can apply Theorem 4.1 for 'Conjecture' in [4].

\section{REMARK}

In this section, we will discuss about the referee comments about Theorems 2.1 and 2.2. Let $E_{2}=\left\{a \in E: \rho_{2}(a)>0\right\}$ and $E_{\infty}=\left\{a \in E: \rho_{\infty}(a)>0\right\}$, and $Z(\psi)=\left\{\lambda \in D^{2}: \psi(\lambda)=0\right\}$ for $\psi$ in $H^{2}\left(D^{2}\right)$. He suggests the following $(1) \sim(5)$. (2) of the comment shows the converse of Theorem 2.1.

(1) There is $\psi$ in $H^{2}\left(D^{2}\right)$ such that $S_{\psi}$ is a nonzero operator of finite rank if and only if $E_{2} \neq \varnothing$ :

If there exists $\psi$ in $H^{2}\left(D^{2}\right)$ which is of finite rank $n \neq 0$ then $E_{2} \neq \varnothing$. This is just Theorem 2.2. However we could not show the converse is true or not. If $\rho_{2}(a)>0$ for some $a \in E$, let $\psi$ be a function in $H^{2}\left(D^{2}\right)$ such that $\psi(a) \neq 0$ and $\psi=0$ on $E \backslash\{a\}$. Then there exists $f$ in $N \cap H^{\infty}\left(D^{2}\right)$ such that $(\psi f)(a) \neq 0$ and $\psi f=0$ on $E \backslash\{a\}$. Hence $\operatorname{dim} S_{\psi}\left(N \cap H^{\infty}\left(D^{2}\right)\right)=1$. But we could not show $S_{\psi}$ is bounded. Hence $S_{\psi}$ may not be of finite rank.

(2) For every $S_{\psi}$ of finite rank there exists a $\phi$ in $H^{\infty}\left(D^{2}\right)$ such that $S_{\psi}=S_{\phi}$ if and only if $E_{2}=E_{\infty}$ : 
The 'if' part is clear by Theorem 2.1 and 2.2. We will show the 'only if' part. If $S_{\psi}$ is of finite rank $n \neq 0$ by the proof of Theorem $2.1|\psi|>0$ on $E \backslash Z(\psi)=\bigcup_{j=1}^{m}\left(a_{j}, b_{j}\right)$. Moreover for each $1 \leq j \leq m$, there exists a $f_{j} \in$ $H^{\infty}\left(D^{2}\right)$ such that $f_{j}\left(a_{j}, b_{j}\right)=1$ and $f_{j}\left(a_{\ell}, b_{\ell}\right)=0$ for $j \neq \ell$. Put $\psi_{j}=f_{j} \psi$ then $\psi_{j}\left(a_{j}, b_{j}\right)=\psi\left(a_{j}, b_{j}\right) \neq 0$ and so $\rho_{2}\left(\left(a_{j}, b_{j}\right)\right)>0$. Since $S_{\psi_{j}}=S_{f_{j}} S_{\psi}$ because $S_{z} S_{\psi}=S_{\psi} S_{z}$ and $S_{w} S_{\psi}=S_{\psi} S_{w}, S_{\psi_{j}}$ is bounded. By hypothesis, there exists $\phi_{j}$ in $H^{\infty}\left(D^{2}\right)$ such that $\psi_{j}-\phi_{j} \in M$ and so $\psi_{j}=\phi_{j}$ on $E$. Therefore $\phi_{j}\left(a_{j}, b_{j}\right) \neq 0$ and $\phi_{j}=0$ on $E \backslash\left(a_{j}, b_{j}\right)$. Hence $\rho_{\infty}\left(\left(a_{j}, b_{j}\right)\right)>0$. Therefore $E_{2}=E_{\infty}$.

(3) $S_{\psi}$ is a nonzero operator of finite rank if and only if $E \backslash Z(\psi)$ is a finite set :

If $S_{\psi}$ is of finite rank $n \neq 0$ then by the proof of Theorem $2.1 \psi=0$ on $E \backslash \bigcup_{j=1}^{m}\left(a_{j}, b_{j}\right)$ and so $E \backslash Z(\psi)$ is a finite set. For the converse, even if $E \backslash Z(\psi)=$ $\left(a_{1}, b_{1}\right)$ by the same reason to $(1)$ we could not show $S_{\psi}$ is of finite rank.

(4) When $S_{\psi}$ is bounded, the rank $n$ of $S_{\psi}$ equals to the number $m$ of elements in $E \backslash Z(\psi)$ :

This is clear for $n=0$. When $n=1$, the proof of Theorem 2.1 and the last three lines of the section 2 show (4) because $S_{\psi}$ is bounded. In general, by the proof of Theorem 2.1, it is easy to see $n \leq m$. However it seems to be $m>n$.

(5) Let $S_{\psi}$ be of finite rank. Then there is a $\phi \in H^{\infty}\left(D^{2}\right)$ such that $S_{\psi}=S_{\phi}$ if and only if $E_{2} \backslash Z(\psi)=E_{\infty} \backslash Z(\psi)$ :

The 'if' part is clear by Theorem 2.1 and 2.2. The 'only if' part is not true. In fact, if $\psi$ is nonzero constant $\alpha$ and $S_{\psi} \neq 0$ then the rank of $S_{\psi}$ is one. On the other hand, $E(\psi)=\varnothing$. In this case, $\phi=\psi$. Of course, there exists $E$ such that $E_{2} \neq E_{\infty}$.

Acknowledgment. The author would like to thank the referee for carefully reading the paper and providing suggestions for improvements.

\section{REFERENCES}

1. J. Agler and J.E. McCarthy, Nevanlinna-Pick interpolation on the bidisc, J. Reine Angew. Math. 506 (1999), 191-204.

2. E. Amar and C. Menini, On an operator theory approach to the corona problem, Bull. London Math. Soc. 34 (2002), 369-373.

3. R.M. Aron, J. Globevnik and M. Schottenloher, Interpolation by vector-valued analytic functions, Rend. Mat. (6) 9 (1976), no. 2, 347-364.

4. T. Nakazi and M. Seto, Double commuting compressed shifts and generalized interpolation in the Hardy space over the bidisk, Integral Equations Operator Theory 56 (2006), 543-558.

5. W. Rudin, Function Theory in Polydiscs, Benjamin, New York, 1969.

6. D. Sarason, Generalized interpolation in $H^{\infty}$, Trans. Amer. Math. Soc. 127 (1967), 179203.

Hokusei Gakuen University, 2-3-1, Ohyachi-Nishi, Atsubetu-ku, Sapporo 0048631, JAPAN.

E-mail address: z00547@hokusei.ac.jp 